\title{
Tuberculosis and Diabetes Mellitus Comorbidity among the Ageing Population: A Threat to the Public Health System of Nepal
}

\author{
Bijay Maharjan, ${ }^{1}$ Hom Nath Chalise,$^{2}$ Mamta Thapa ${ }^{3}$ \\ 1Japan-Nepal Health and Tuberculosis Research Association, Kathmandu, Nepal, 2Population Association of \\ Nepal, Kathmandu, Nepal, ${ }^{3}$ Asian College for Advance Studies, Kathmandu, Nepal.
}

\begin{abstract}
Ageing population is attributable to the growing trend of diabetes mellitus. Diabetic patients are three times greater risk of developing tuberculosis. The review aims to describe the state of tuberculosis and diabetes mellitus comorbidity, risk factors for the comorbidity from the perspective of the ageing in Nepal. It is a systematic narrative review of literature in Google Scholar and Pubmed. At first, title and abstract of an article was reviewed for relevance, and then full article was reviewed for validity. The secondary data was retrieved from the Central Bureau of Statistics of Nepal and WHO, and analysed in the Ms-Excel. In Nepal, the population is ageing. The risk factors associated with diabetes are escalating. On the other hand, tuberculosis is endemic and about $45 \%$ of the Nepalese people are infected with tuberculosis bacteria. These circumstances have laid the foundation that fosters tuberculosis and diabetes co-epidemic in the future. The clinical management of patients with the comorbid condition is a difficult task because diabetes and tuberculosis interact with each other, one worsening the other. The upsurge of the co-morbidity needs the provision of more health services threatening the public health system of Nepal. It is fundamental to create a mechanism to integrate diabetes and tuberculosis program such as screening, diagnosis and management of the both diseases at the all levels of health service delivery. Furthermore, increase awareness of healthy lifestyle and the prevention of the risk factors for tuberculosis and diabetes could reduce the occurrence of the comorbidity in the future.

Keywords: Ageing population; co-morbidity; diabetes mellitus; Nepal; tuberculosis.
\end{abstract}

\section{INTRODUCTION}

The ageing population is increasing in most countries and poses several public health challenges. ${ }^{1}$ Risk of diabetes mellitus (DM) rises with an increase in age. DM intensifies the risk of developing active tuberculosis (TB) by 3 times. ${ }^{2,3}$ Ageing and diabetes confine the immunity which transforms the latent TB to active TB. ${ }^{4}$ Globally, $15 \%$ of all TB cases are attributable to diabetes. ${ }^{5}$ The incidence rate of TB and diabetes co-morbidity is rising. ${ }^{6}$

In Nepal, the ageing population and prevalence of diabetes is increasing. ${ }^{7,8}$ Almost half of the population is infected with TB. ${ }^{9}$ TB among the ageing population has been a rising trend in Nepal. ${ }^{10} \mathrm{It}$ has shaped a conducive environment to propagate tuberculosis and diabetes mellitus (TB-DM) comorbidity. The review explores the influence of ageing population in the progression of TB-DM comorbidity and assesses the status of the comorbidity, related risk factors and its consequences for the health system in Nepal.

\section{METHODS}

The study is a systematic narrative assessment of literature and data sources. We searched the PubMed and Google Scholar database by use of the following search terms: ageing, tuberculosis, diabetes mellitus, TB and diabetes comorbidity and Nepal published in English regardless of year of study. Manual searched references were also included to prepare this article. Figure 1 shows the process of selection of the articles in the study. At first, the title and abstract of the was reviewed for relevance and then full article was reviewed for valid methods and results. The full articles with valid methods and the results were included in the study. Secondary data were obtained from Central Bureau of Statistics and World Health Organization (WHO) website. The analysis of the secondary data was carried out using rate, proportion, and future estimation was done using the exponential and log transformation in Microsoft-Excel version 16
DOI: http://dx.doi.org/10.3126/ inhrc.v16i2.20294
Correspondence: Mr Bijay Maharian, Japan-Nepal Health and Tuberculosis Research Association, Subidanagar, Koteshwor, Kathmandu, Nepal. Email: bjmaharjan@hotmail.com, Phone: +977 9849554444. 


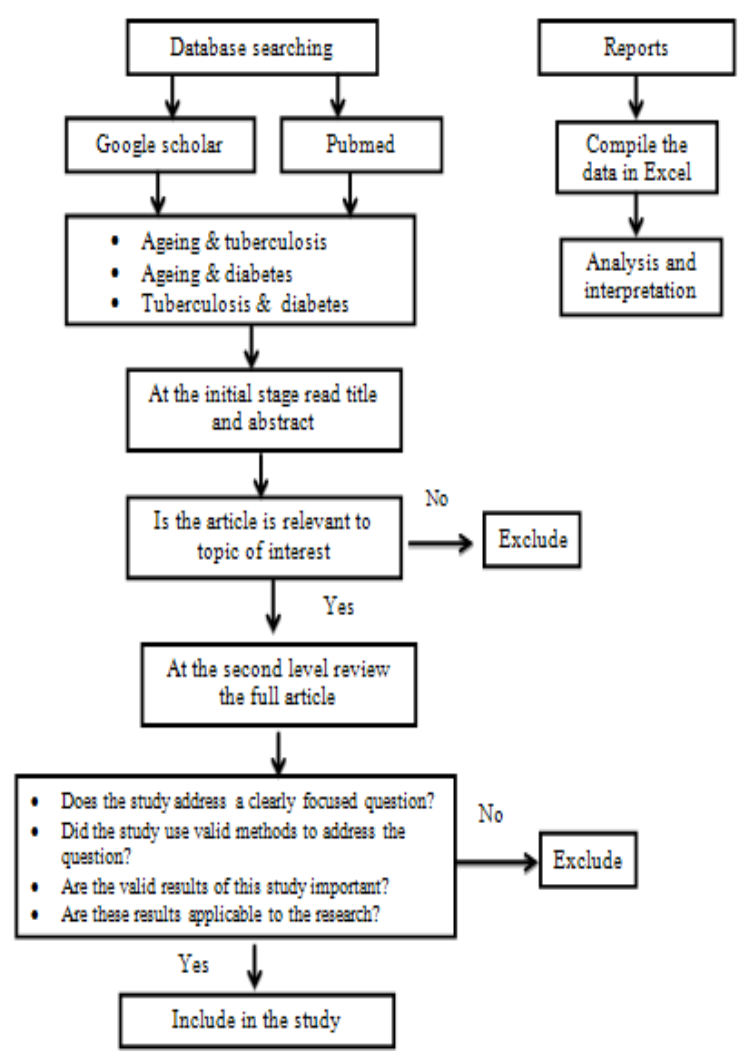

Figure 1. Flowchart for the process of selection of literatures.

\section{AGEING POPULATION IN NEPAL}

According to the Nepal Elderly Act 2000, individuals 60 years and above are considered as elderly. There are very few studies conducted among ageing population in Nepal. ${ }^{11,} 12$

The size and growth rate of the elderly population has increased in the past 6 decades. The total population of the country has amplified by 220 percent, while the total elderly population has increased by 4.25 percent from $1952 / 1954$ to 2011 . The elderly population will be double in 19 years if the elderly population growth rate continues to surge at the current rate, indicating to reach 4.3 million by $2030 .{ }^{13}$ It indicates the growth rate of the elderly is persistently higher than that of the total population. Moreover, due to the rapid increase in life expectancy at birth in Nepal, the elderly population in the future would appear to escalate at an even quicker pace. Census reports reveal the median age of Nepal increased to 23 years and ageing index increased to 23.3 years. ${ }^{14}$ It shows the population of Nepal is gradually ageing.

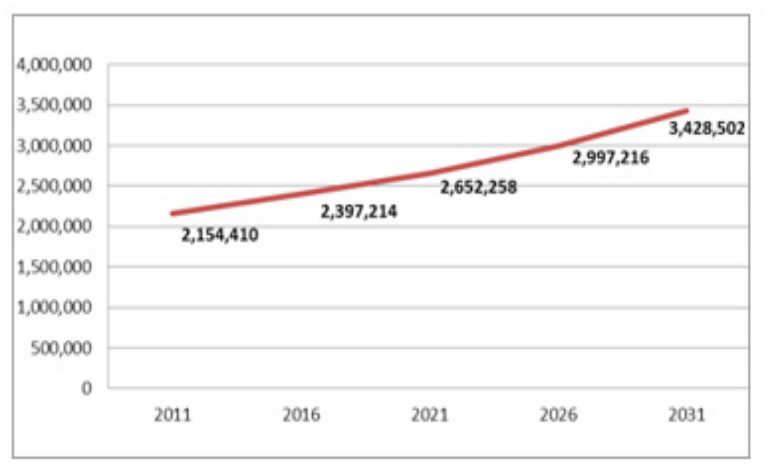

Figure 2. Increasing trend of population over 60 years of age in Nepal.

It has been documented that an ageing population can have profound implications on the occurrence of communicable and non communicable diseases. Likewise, the ageing population is risk factor for both tuberculosis and diabetes. ${ }^{15,16}$ The presence of risk factors such as tobacco use, low socioeconomic status and past history of disease, and adverse reactions during treatment make them more susceptible for the both diseases. At the same time, social marginalization, reduced mobility and financial dependency often discouraged elder population from seeking health care resulting under-diagnosis among older people and are more likely to die unscreened and untreated. ${ }^{17-19}$

\section{Table 1. Situation of elderly population growth in Nepal. ${ }^{13}$}

\begin{tabular}{|c|c|c|c|c|c|}
\hline \multirow{2}{*}{ Census Year } & \multirow{2}{*}{ Elderly Population } & \multicolumn{2}{|c|}{ Population Growth Rate } & \multirow{2}{*}{60 years and older $\%$} & \multirow{2}{*}{ Elderly Sex Ratio } \\
\hline & & Total & Elderly & & \\
\hline $1952 / 54$ & 409,761 & - & - & 5.0 & 80.2 \\
\hline 1961 & 489,346 & 1.65 & 1.79 & 5.2 & 83.3 \\
\hline 1971 & 621,597 & 2.07 & 2.42 & 5.4 & 92.2 \\
\hline 1981 & 857,061 & 2.66 & 3.26 & 5.7 & 111.1 \\
\hline 1991 & $1,071,234$ & 2.10 & 2.26 & 5.8 & 102.7 \\
\hline 2001 & $1,504,311$ & 2.24 & 3.40 & 6.5 & 101.7 \\
\hline 2011 & 2154410 & 1.35 & 3.59 & 8.13 & 97.75 \\
\hline
\end{tabular}




\section{TUBERCULOSIS IN NEPAL}

Tuberculosis remains endemic in Nepal. Despite the continuous efforts, there is a low rate of decline in annual TB incidence rate. ${ }^{20}$ There were an estimated 59,000 prevalent cases with an incidence rate of 154 per 100,000 population and 31,764 cases were notified in 2017. ${ }^{21,22}$ Among these cases, about $71 \%$ were pulmonary cases out of which $77 \%$ were bacteriologically confirmed. The treatment success rate is about $92 \%$. Males were nearly 1.8 times more than females with reported TB case. $^{21}$

The majority of the TB burden is in the active population (15-44) age group. The childhood TB was around 5.6\%.21 The trend in these age group has remained identical for over the years. However, the burden of TB among 65 years and above population is rising irrespective in the total number of notified cases in Nepal. ${ }^{21}$ The most probable reasons for continuous growth in tuberculosis cases among older population; a rise in the proportion of an ageing population, proliferation of risk factors such as diabetes or perhaps both. TB diagnosis and treatment is problematic in elderly due to occurrence of acute or chronic diseases and weakened immune defense mechanism. ${ }^{23}$ In addition, TB treatment among the elder population is challenging because of the amplified incidence of adverse drug reactions. ${ }^{24}$ Therefore, TB in elderly is regarded as public health challenge. ${ }^{24}$

\section{DIABETES AND ITS RISK FACTORS IN NEPAL}

It was estimated, there were 526,000 cases of diabetes in 2015 with the prevalence of $9.1 \%$ in Nepal. ${ }^{25,26}$ One third of diabetes patients remain undiagnosed. ${ }^{27}$ The pooled prevalence of diabetes was $8.4 \%$, ranging from a minimum of $1.4 \%$ to a maximum of $19.0 \% .28$ The prevalence of diabetes in urban areas among aged over 20 years is $14.6 \%$ and it upsurges to $19 \%$ among aged 40 years and older while in the rural areas, the prevalence was $2.5 \%{ }^{29}$ The prevalence of diabetes in urban and rural populations was $8.1 \%$ and $1.0 \%$, respectively. ${ }^{28}$ The prevalence rate of diabetes constantly indicated a rise in prevalence with increasing age. ${ }^{26,30}$ The odds ratio for increasing age was 1.05 (95\% Cl: 1.04-1.05). ${ }^{28}$ Diabetes was one of the major cause of morbidity in the elderly population. ${ }^{31}$ Ageing of the population was accountable for $19 \%$ of the progress of diabetes mellitus, rise in the age-specific prevalence of diabetes was attributed by $81 \%{ }^{32}$

Diabetes was slightly higher among male $(10.5 \%)$ than female $(7.9 \%)$. The prevalence of overweight was $16.70 \%$. Similarly, the prevalence of obesity is $2.9 \%$
(Table 2). The prevalence of physical inactivity was $3.3 \% .^{33}$ The trend analysis of the metabolic risk factors in Nepal between 1980 and 2008 illustrates high blood pressure, body mass index and blood glucose level as major risk factors for diabetes. ${ }^{33} \mathrm{~A}$ survey showed that a family history of diabetes was present in about one-third of the patients. The highest prevalence of diabetes was seen among the elderly (25.9\%). Among those elderly, $17.3 \%$ were newly diagnosed and $8.6 \%$ took diabetes medication. ${ }^{34}$

\begin{tabular}{lrrr}
$\begin{array}{l}\text { Table 2. Prevalence of diabetes and related risk } \\
\text { factors in Nepal. }{ }^{25}\end{array}$ & Male & Female & Total \\
$\begin{array}{l}\text { Risk factors for } \\
\text { diabetes }\end{array}$ & $10.50 \%$ & $7.90 \%$ & $9.10 \%$ \\
\hline Diabetes & $13.60 \%$ & $19.60 \%$ & $16.70 \%$ \\
\hline Overweight & $1.70 \%$ & $4.10 \%$ & $2.90 \%$ \\
\hline Obesity & $3.90 \%$ & $2.80 \%$ & $3.30 \%$ \\
\hline Physical inactivity & & & \\
\hline
\end{tabular}

\section{TUBERCULOSIS AND DIABETES CO-MORBIDITY IN NEPAL}

TB-DM comorbidity is an imminent epidemic. ${ }^{35-37}$ The burden of TB-DM co-morbidity was more prominent in Nepal as the occurrence of diabetes is rising and TB is endemic. ${ }^{38}$

About $8 \%$ of diabetic patients had pulmonary tuberculosis in Nepal. ${ }^{39}$ More than nine percent of TB patients had diabetes mellitus, among them nearly seven percent were self- reported cases and more than $2 \%$ were identified by the lab test among the suspected diabetes cases. Among the total male TB patient $11.8 \%$ had diabetes mellitus, while more than $8 \%$ of the female TB patients had diabetes mellitus. Nearly $38 \%$ of the TB and diabetes comorbidity was prevalent in 45-55 aged groups. Similarly, $33 \%$ of the comorbidity were among the aged group of more than 55 years. It indicated that TB and diabetes comorbidity was higher in older adults and the ageing population, one fourth of TB-diabetes cases in the 35-44 aged groups. ${ }^{40}$

The risk factors of comorbidity were older age, tobacco use, high income status and history of high blood pressure. ${ }^{40}$ Nepal is also facing the consequences of sedentary lifestyle leading obesity and metabolic syndrome. ${ }^{41}$ Studies had shown the prevalence of overweight and obesity in certain sections of the population to be as high as $32.9 \%$ and $7.2 \%$ respectively. The combined risk factors for non-communicable diseases were increased including diabetes and age. ${ }^{42}$ 


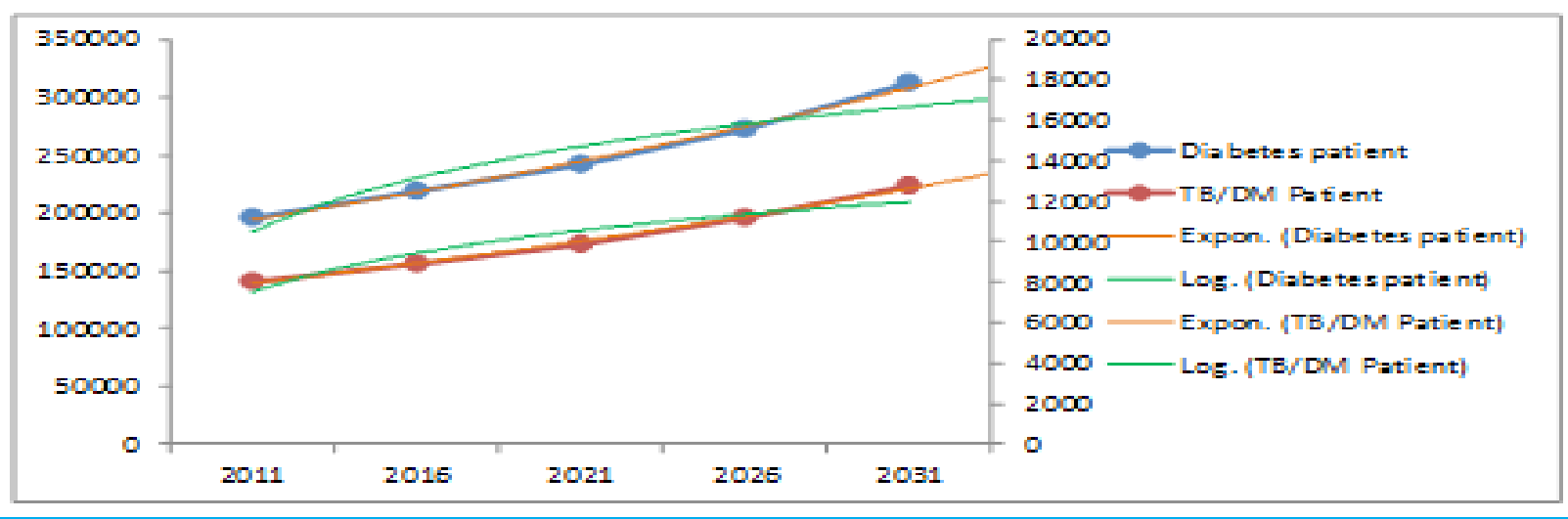

Figure 3. Estimation of number of diabetes and TB/Diabetes patient over the 60 years in Nepal.

Figure 3 reveals that, increasing trend of diabetes patient over the years. As the population in over the 60 years age group is increasing (Figure 2). Along with that there is increasing trend of TB and diabetes patient over the years in the age group of over 60 years. It means each year increase in TB patient with diabetes.

\section{DISCUSSION}

This article revealed that there has been a growing burden of diabetes due to change in age structure of the population. There is a rise in risk factors for diabetes such as unhealthy dietary pattern, obesity, reduced physical activities in Nepal. Despite of the nationwide coverage of DOTS at health institutions, TB incidence has not decreased in Nepal. The affected age group by TB is shifting to the elderly population in Nepal. There are complications and constraints on finding and treating TB cases in the aged populations since diabetes have a high prevalence among these populations. TB-DM comorbidity is changing the landscape of TB care and prevention, especially among the ageing population. The inclining trend of tuberculosis among the elderly population could be attributed to increases in the ageing population or risk factors such as diabetes or perhaps, the occurrence of both.

Literature shows that diabetes is an important risk factor for the development of TB and affect disease manifestation and treatment response. ${ }^{43}$ It intensifies the time to sputum culture conversion, and increases the risk of death and TB relapse. However, there is no evidence that diabetes increases the risk of recurrence caused by drug-resistant strains ${ }^{57}$ It suggests that more relapse TB patient in the coming years among diabetes patients. However, there is the need for scientific research on relapse rate of $\mathrm{TB}$ among old age population and its linkage to diabetes.
Our study indicates that there will be more old age population than before in Nepal. The review designates, the majority of these populations reside in the urban areas where there are favourable conditions for TB transmission like overcrowding, development of slum areas, mobile population, unorganized settlement, harmful working conditions. There is high prevalence of tuberculosis in urban areas. There is the rapid growth of urban areas in Nepal and the prevalence of diabetes is significantly higher in the urban areas. This indicates there is a higher probability of increasing incidence of tuberculosis attributable to diabetes mellitus in the urban areas.

We have attempted to review the correlation of TB-DM comorbidity with age group. Our study shows that the prevalence of TB and diabetes comorbidity is higher among the older ageing population. A study reveals that patients in pulmonary tuberculosis and diabetes group were significantly older $(53.34 \pm 14.06$ year $)$ in comparison to their non-diabetic counterparts (44.35 \pm 18.14 year). ${ }^{44}$ A study conducted in India demonstrated that young patients with diabetes was at a higher risk of acquiring tuberculosis, this finding varies from our study and the relative risk was higher in countries with a higher incidence of tuberculosis, suggesting that a similar prevalence of diabetes mellitus might contribute to the tuberculosis epidemic in developing countries. ${ }^{45}$ It means more scientific evidence is necessary on age group variation in risk of TB and diabetes comorbidity.

There are more male TB patients than females TB patient. ${ }^{46}$ Similarly, there are more male diabetes patients than female. ${ }^{47}$ The prevalence of TB-DM comorbidity is higher among male than female. It is more likely that males are more risk of developing the comorbidity. It suggests male is at higher risk of developing TB than female. It is necessary to carry out more studies in TB and diabetes comorbidity with a gender perspective. 
A study was conducted on screening, TB patients for diabetes in India reveals that males with TB are more likely to have diabetes than females. ${ }^{45}$

The study illustrates a resilient relationship between changing age structure, diabetes and tuberculosis. The increase in diabetes patient could increase TB and pose a great challenge to the end TB strategy by increasing the risk of TB as well as the severity of the disease. So, to achieve national goals, including end TB strategy, the National Tuberculosis Programme should develop protocols, promote research in TB/diabetes especially focusing in the ageing population and coordinate and collaborate for the integrated TB diabetes programme like screening of diabetes mellitus among TB patients, also screening of tuberculosis among the diabetes patients, establish the referral mechanism between TB services and diabetes services, and execute the integrated TB/diabetes activities at the community and health institutional level.

Implications of the tuberculosis and diabetes comorbidity for the public health system in Nepal

The clinical management of patients with the comorbid condition is very difficult. ${ }^{48}$ Diabetes is linked with failure to sputum conversion and treatment failure that could increase in MDR TB patients. ${ }^{49,50}$ Relapse TB is highly associated with the development of MDR TB. ${ }^{51} \mathrm{It}$ is estimated that $13 \%$ growth in diabetes prevalence possibly will reduce the shrink in tuberculosis incidence by $8 \%$ or more. ${ }^{49,52}$ People with diabetes who are diagnosed with TB have a higher risk of death during TB treatment. It reveals diabetes prolongs the duration of TB treatment which increases the catastrophic economic burden to the patient and family members. ${ }^{53}$ Therefore, the rise in the prevalence of diabetes could uphill tuberculosis incidence, including MDR and act as a barrier to achieve goals of the end TB strategy. On the other hand, TB often effects diabetes causing hyperglycemia and can temporarily cause impaired glucose tolerance, which is a risk factor for developing diabetes. ${ }^{53}$ Diabetes is complicated by the presence of TB.It is difficult to manage diabetes among TB patients. Furthermore, some of the anti-TB drugs (particularly rifampicin) make the situation sometime worsen to control diabetes. ${ }^{54,55}$ This kind of problem arises when anti-TB (especially rifampicin and isoniazid) and oral anti-diabetic drugs, interact with each other. ${ }^{55,56}$ Therefore, the merging of the two diseases is fatal. It poses a threat to the entire public health system because the number of comorbid conditions is increasing, affecting a larger section of the population across all the communities.
From a health system perspective, Nepal's health care shortfalls are largely attributed to low government spending, unevenly distributed health services, limited affordability, inadequate supply of essential drugs, low awareness of the disease and possible treatment, including the availability of limited health workforce. ${ }^{57}$ In Nepal, there is only one endocrinologist per 1000000 populations. ${ }^{58}$ There are several challenges in diabetes management including the high cost of treatment, limited health care facilities, and lack of disease awareness among patients. No specific guideline was identified for the prevention and treatment of diabetes in Nepal. ${ }^{59}$ There was no established mechanism of collaboration for TB and diabetes activities, detect and manage TB in patients with diabetes, detect and manage diabetes in a patient with TB. Ironically senior citizens comprise the age group that has more specific demands such as the support for health services, income, care, and moral and physical support. The issue is very critical as a proportion of the older population in Nepal grows amidst lack of a comprehensive health care system, social security system, undeveloped pension system and a stagnant economy. ${ }^{60}$ Despite there is an increasingly ageing population and risk factors among these populations; little attention has been given to the TB among old age population in Nepal. Although currently underappreciated, tuberculosis among older adults will pose major challenges to National Tuberculosis Programme (NTP) especially comorbidity of diabetes in the future.

It is essential to establish a mechanism to integrate diabetes and TB program at the national, provincial, local and the community level. It is proposed to initiate screening and management of latent TB infection among the ageing population and diabetes. Likewise, persuade screening of tuberculosis among the diabetes patients as well as initiate screening of diabetes among the TB patients among ageing population. Likewise, NTP have to explore the possibility to explore the adapting diabetes services including its monitoring, treatment outcome and reporting in the DOTS system. Furthermore, it is equally important to prioritize the interventions on health literacy and behaviour change communication aimed on primordial prevention for healthy lifestyle across all the communities as well as increase awareness on the prevention of the risk factors for TB and diabetes mellitus comorbidity.

\section{Limitation of the study}

Most of the TB-DM comorbidity studies focus on the correlation of TB and diabetes of the general population. However, there are the limited and different age groups 
in the literature of these two conditions. We were not able to standardize and compare the data.

It is suggested to the future researcher in TB and diabetes to use standardized age group. So that it will be easier and valid for the reviewers and researchers. The treatment outcome among the diabetic and aged population should also be understood. The complications of TB diabetic treatment and morbidity, mortality need to be examined. It is necessary of statistical analysis for the age-wise distribution of types of TB and its trend and type of TB among the diabetic patients.

\section{CONCLUSIONS}

The prevalence of diabetes mellitus is in increasing trend, especially among the old age population. On the other hand, the incidence of tuberculosis has not decreased in Nepal even though the nationwide coverage of the DOTS. The case notification rate of TB has remained stagnant in other age groups, but it is an increasing trend among the older age groups in Nepal. The demographic transition leading large proportion of the ageing population along with the escalation in diabetes has laid the foundation that fosters TB and diabetic co-epidemic in the near future. The higher incidence of co-morbidity calls for the provision of more health services as well as altering the package of services to better treat and manage the diseases of old age.

It is crucial to create a mechanism to integrate diabetes and TB program at the national, provincial, local and community level. Implementation of interventions such as screening and management of latent TB infection among the ageing population and diabetes. Likewise, NTP can explore the prospect to integrate diabetes services in DOTS system. Furthermore, executing the interventions on primordial prevention for healthy lifestyle across all the communities as well as increase awareness on the prevention of the risk factors for TBDM comorbidity could reduce the occurrence of TB-DM comorbidity and its management.

\section{ACKNOWLEDGMENTS}

We are thankful to Ram Sharan Gopali of Japan-Nepal Health and Tuberculosis Research Association for helping in developing the concept and motivate to write the paper and Ashok Pandey of Nepal Health Research Council for sharing the information related to the process of publications and kind support during our study period.

\section{REFERENCES}

1. United Nations. World population ageing [Internet]. Department of Economic and Social Affairs, Population Division; 2015 [cited 2017 Mar 30]. [Full Text]

2. Kirkman, MS. Diabetes in older adults [Internet]. Diabetes Care. 2012 [cited 2017 Mar 26]. [Link]

3. WHO.TB comorbidities and risk factors [Internet]. WHO. 2017 [cited 2017 Mar 30]. [Link]

4. Menon S, Rossi R, Nshimyumukiza L, Wusiman A, Zdraveska N, Eldin MS. Convergence of a diabetes mellitus, protein energy malnutrition, and TB epidemic: the neglected elderly population. BMC Infect Dis [Internet]. 2016 Dec [cited 2017 Apr 11];16(1). [Link]

5. Zheng $\mathrm{C}, \mathrm{Hu}$ M, Gao F. Diabetes and pulmonary tuberculosis: a global overview with special focus on the situation in Asian countries with high TB-DM burden. Global Health Action. 2017 Jan;10(1):1264702.

6. Worrall JS. Addressing the double-burden of diabetes and tuberculosis: lessons from Kyrgyzstan [Internet]. Globalization and Health. 2017 [cited 2017 Mar 27]. [Link]

7. Singh ML. Ageing of the Population of Nepal. Population Monograph of Nepal. 2003;2:251-294.

8. Nepal Health Research Council. Noncommunicable diseases risk factors: STEPS survey Nepal [Internet]. Nepal: NHRC; 2013 [cited 2016 May 18].[Full Text]

9. National Tuberculosis Centre. Nepal's Tuberculosis Scenario [Internet]. National Tuberculosis Centre. 2017 [cited 2017 Apr 4]. [Full Texxt]

10. National Tuberculosis Centre. National Tuberculosis Programme: Annual Report 2014. Thimi, Bhaktaour: National Tuberculosis Centre; 2014.

11. Nepal law commission. Senior Citizens Act, 2063 [Internet]. 2006 [cited 2017 Sep 24]. [Full Text]

12. Chalise HN, Saito T, Kai I. Self-reported health: A study of older adults from a developing country-Nepal. Bioscience Trends. 2007 Oct 1;1:102-7.

13. Chalise, H N, Lamsal U. Walking and sleep quality of Nepalese elderly living in Briddaashram, A research report submitted to University Grant Commission, Bhaktapur. 2013.

14. Central Bureau Statistics. Population monograph of Nepal. Kathmandu: Government of Nepal, National Planning Commission Secretariat, Central Bureau of Statistics; 2014. 3 p. 
15. Byng-Maddick R, Noursadeghi M. Does tuberculosis threaten our ageing populations? BMC Infect Dis [Internet]. 2016 Mar 11 [cited 2018 Jan 7];16. [PubMed]

16. Suastika K, Dwipayana P, Semadi MS, Kuswardhani RAT. Age is an important risk factor for type 2 diabetes mellitus and cardiovascular diseases. IntechOpen. 2012. [cited 2018 Jan 7]; [Full Text]

17. World Health Organization. Factsheet: a Collaborative framework for care and control of tuberculosis and diabetes [Internet]. WHO; 2016 [cited 2017 Mar 30]. [Link]

18. Katha A J. Old age hits tuberculosis [Internet]. Times of India. 2013 [cited 2017 Sep 24]. [Full Text]

19. Negin J, Abimbola S, Marais BJ. Tuberculosis among older adults - time to take notice. Int J Infect Dis. 2015;32:1357.

20. National Tuberculosis Centre. TB factsheet [Internet]. National Tuberculosis Centre. 2017 [cited 2017 Apr 11]. [Full Text]

21. National Tuberculosis Centre. NTP Annual Report 2017. Bhaktapur: National Tuberculosis Centre; 2017.

22. World Health Organization. Tuberculosis profile of Nepal [Internet]. 2016 [cited 2018 Jan 3]. [Full Text]

23. Donald PR, Marais BJ, Barry CE. Age and the epidemiology and pathogenesis of tuberculosis. The Lancet [Internet]. 2010 May [cited 2017 Jul 15];375(9729):1852-4. [Full Text]

24. Rajagopalan S, Yoshikawa TT. Tuberculosis in the elderly. J Gereontol Geriatr Res. 2000;33(5):374-80.

25. World Health Organization. Diabetes country profile of Nepal [Internet]. Geneva, Switzerland: WHO; 2015 [cited 2016 Jun 21].[Full Text]

26. International Diabetes Federation. Nepal [Internet]. International Diabetes Federation. 2015 [cited 2017 Apr 2]. [Full Text]

27. Shrestha PK. Prevalence of type 2 diabetes mellitus [Internet]. Diabetes Nepal Conference; 2014 [cited 2017 Apr 12]; Kathmandu. [Full Text]

28. Gyawali B, Sharma R, Neupane D, Mishra SR, van Teijlingen E, Kallestrup P. Prevalence of type 2 diabetes in Nepal: a systematic review and meta-analysis from 2000 to 2014. Global health action [Internet]. 2015 Nov 26 [cited 2017 Apr 2];8. [PubMed]

29. Singh DL, Bhattarai MD. High prevalence of diabetes and impaired fasting glycaemia in urban Nepal. Diabetic Med. 2003 Feb;20(2):170-1.

30. Mehta KD, Karki P, Lamsal M, Paudel IS, Majhi S, Das BKL, et al. Hyperglycemia, glucose intolerance, hypertension and socioeconomic position in eastern Nepal. Southeast Asian JTrop Med Public Health. 2011 Jan;42(1):197-207.

31. Tessier D, Avila-Funes JA, Aguilar-Navarro S. [Diabetes and changes in functional status of the elderly: a reality?]. Revista de investigacionclinica.Hospital de enfermedads de la nutricion 2010 Aug;62(4):318-22.

32. Sudharsanan N, Ali MK, Mehta NK, Narayan KMV. Population ageing, macroeconomic changes, and global diabetes prevalence, 1990-2008. Population Health Metrics [Internet]. 2015 Dec [cited 2017 Apr 3];13(1). [Link]

33. Gautam R. NCDs in Nepal: burgeoning burden amid low priority and the ways forward. Health Prospect [Internet]. 2013 [cited 2017 Apr 2];11. [Full Text]

34. Chhetri MR, Chapman RS. Prevalence and determinants of diabetes among the elderly population in the Kathmandu Valley of Nepal. Nepal Med Coll J. 2009 Mar;11(1):34-8.

35. World Diabetes Foundation, The Union. The looming coepidemic of TB-Diabetes: A call for action [Internet]. 2015 [cited 2016 Apr 19]. [Full Text]

36. Cousins S. Global health experts warn of a "looming coepidemic" of tuberculosis and diabetes. BMJ. 2015 Nov 3;h5912.

37. World Diabetes Foundation. Bali declaration calls for action against TB-diabetes co-epidemic [Internet]. News. 2015 [cited 2017 Sep 24]. [Full Text]

38. Baghaei P, Marjani M, Javanmard P, Tabarsi P, Masjedi MR. Diabetes mellitus and tuberculosis facts and controversies. J Diabetes Metab Disord. 2013 Dec 20;12:58.

39. Regmi HS, Gurung R, Sharma SK, Pradhan B, Bhattacharya SK. Pulmonary tuberculosis among diabetic patients in Dharan Municipality, Eastern Nepal. Int J Inect Dis. 2014 Apr;21:304.

40. Thapa B, Paudel R, Thapa P, Shrestha A, Poudyal A. Prevalence of diabetes among tuberculosis patients and associated risk factors in Kathmandu Valley. SAARC Journal of tuberculosis, lung disease and HIVAIDS. 2016 Oct $25 ; 12(2): 20$.

41. Vaidya AK, Pokharel PK, Nagesh S, Karki P, Kumar S, Majhi S. Association of obesity and physical activity in adult males of Dharan, Nepal. Kathmandu Univ Med J. 2006 Jun;4(2):192-7.

42. Nepal Health Research Council. Non Communicable Disease Risk Factors: STEPS Survey Nepal [Internet]. Kathmandu: Nepal Health Research Council; 2013 [cited 2017 Apr 3]. [Full Text] 
43. World Health Organization, International Union against Tuberculosis and Lung Disease. Collaborative framework for care and control of tuberculosis and diabetes [Internet]. Geneva:World Health Organization; 2011 [cited 2016 Aug 3]. [Full Text]

44. Rawat J, Biswas D, Sindhwani G. Effect of age on presentation with diabetes: Comparison of nondiabetic patients with new smear-positive pulmonary tuberculosis patients. Lung India. 2011;28(3):187.

45. Muruganathan A, Viswanathan V. Section 5 Diabetology. [cited 2017 Apr 4]; [Full Text]

46. Neyrolles O, Quintana ML. Sexual Inequality in Tuberculosis. PLoS Med. 2009 Dec 22;6(12):e1000199.

47. Fennell D. Men at higher risk for type 2 diabetes [Internet]. Diabetes self-management. [cited 2017 Sep 24]. [Link]

48. Riza AL, Pearson F, Ugarte-Gil C, Alisjahbana B, van de Vijver S, Panduru NM, et al. Clinical management of concurrent diabetes and tuberculosis and the implications for patient services. Lancet Diabetes Endocrinol [Internet]. 2014 Sep [cited 2017 Sep 23];2(9):740-53. [Full Text]

49. The Lancet Diabetes Endocrinology. Diabetes and tuberculosis--a wake-up call. Lancet Diabetes Endocrinol. 2014 Sep;2(9):677.

50. Mi F, Tan S, Liang L, Harries AD, Hinderaker SG, Lin Y, et al. Diabetes mellitus and tuberculosis: pattern of tuberculosis, two-month smear conversion and treatment outcomes in Guangzhou, China. Trop Med Int Health. 2013 Nov; 18(11):1379-85.

51. Lee P-H, Lin H-C, Huang AS-E, Wei S-H, Lai M-S, Lin H-H. Diabetes and Risk of Tuberculosis Relapse: Nationwide Nested Case-Control Study. PLoS ONE [Internet]. 2014 Mar 24 [cited 2017 Apr 2];9(3). [PubMed]
52. Pan S-C, Ku C-C, Kao D, Ezzati M, Fang C-T, Lin H-H. Effect of diabetes on tuberculosis control in 13 countries with high tuberculosis: a modelling study. Lancet Diabetes Endocrinol. 2015 May;3(5):323-30.

53. WHO. Factsheet: Collaborative framework for care and control of Tuberculosis and Diabetes [Internet]. WHO; 2016 [cited 2017 Mar 30]. [Link]

54. Siddiqui A. Role of diabetes in prevalence of tuberculosis. J Diabetes Metab Disord [Internet]. 2012 [cited 2017 Sep 24];02(09). [Full Text]

55. Blaschke TF, Skinner MH. The clinical pharmacokinetics of rifabutin. Clinal Infect Dis Off Publ Infect Dis Soc Am. 1996 Apr;22Suppl 1:S15-21; discussion S21-22.

56. Pal R, Ansari MA, Hameed S, Fatima Z. Diabetes mellitus as hub for tuberculosis infection: A Snapshot. Int J Chronic Dis. 2016;2016:1-7.

57. Dhitali SM, Arjun K. Dealing with the burden of hypertension in Nepal: current status, challenges and health system issues. In: Regional Health Forum [Internet]. 2013 [cited 2017 Apr 4]. p. 44-52. [Link]

58. The Himalayan Times. Nepal at high risk of diabetes. Rastriya Samachar Samiti.[Internet]. 2016 Apr 6 [cited 2018 Jan 3];[Full Text]

59. Gyawali B, Ferrario A, Teijlingen VE, Kallestrup P. Challenges in diabetes mellitus type 2 management in Nepal: a literature review. Global Health Action. 2016 Dec;9(1):31704.

60. Limbu A. Age Structure Transition and Senior Citizens in Nepal: The Impending Challenges. Policy [Internet]. 2012 [cited 2017 Mar 31];2012.[Full Text] 